\title{
A Novel Apparatus for Surveillance of Green Energy System Based on WSSs
}

\author{
Joy I.-Z. Chen ${ }^{1 *}$, Yue Hsin Gien ${ }^{1}$, Wen Bin Wu ${ }^{2}$, Chieh Wen Liou ${ }^{2}$ \\ ${ }^{1}$ Department of Electrical Engineering, Dayeh University, Changhua, Chinese Taipei \\ ${ }^{2}$ Department of Electrical Engineering, Asia-Pacific Institute of Creativity, Miaoli, Chinese Taipei

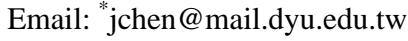

Received October 8, 2012; revised November 9, 2012; accepted November 20, 2012

\begin{abstract}
The WSSs (wireless sensor systems) concept is applied to implement an uninterrupted solar energy surveillance system in this work. The completed system is comprised of three major sub-systems that include a charging sub-system, a control sub-system and a display sub-system. Based on several transmission standards, including Bluetooth, Wifi and Zigbee capability combined with wireless transmission techniques, the proposed surveillance system is designed for monitoring a solar energy system. The performance of the simulated WSSs is evaluated using statistical report results. The proposed surveillance system can be fully extended to several different kinds of applications, such as, health care and environmental inspection. The experimental measurement results significantly show that channel fading over the propagation channel dominates the developed system performance.
\end{abstract}

Keywords: Bluetooth; Wifi; Health Care; Solar Surveillance; WSSs; Zigbee

\section{Introduction}

WSSs (wireless sensor systems) have recently acquired an important role based on several real world application advantages such as low cost, easy establishment, selforganizing capacity and wide deployment for experimental research or real world practical applications. WSSs infrastructure has spread widely in various human life applications such as health care, automotive control, military command, communications and surveillance [1, 2]. Thus, study of the issues in each WSS protocol layer has gradually become a new research trend. These research areas include power consumption networking topology, signal processing, environmental monitoring deployment, transmission Media, etc. It is known that the greater the number of sensor nodes in the network the more precise the results provided to the BS (base station). However, in order to reduce the number of parameters for system performance, decreasing the number of sensor nodes to the optimal number is a good method. The mobility sensors is also an important point that can be applied to solve the coverage hole problem in WSSs $[3,4]$. Combining WNSs with RFID (radio frequency identification) devices is another recent study issue. For example, they can be combined together to solve surveillance issues $[5,6]$. Traditionally, wired transmission is the normal technique applied in energy monitoring. Wired com-

"Corresponding author. munication was applied in monitoring the solar energy with project results reported in $[7,8]$. The system parameters in solar energy systems include the current, voltage and photovoltaic transformation power. Numerous publications have addressed these issues. Authors in [8] proposed a monitoring system design based on solar energy for a greenhouse wireless sensor network. The electrical power usage of facility electrical devices was periodically obtained from each of the wireless sensor devices in [9]. In [10] the authors present an overview of the different energy harvesting technologies and the energy saving mechanisms for WSS. The related research issues on energy efficiency for sensor networks using energy harvesting technology are then discussed. The authors' claimed that the system combined energy management with energy transfer, ensuring the energy collected by the solar energy batteries would be used reasonably. Photovoltaic systems with managed output and methods for managing output variability from photovoltaic systems are described in [11]. A plurality of the photovoltaic modules was configured to receive and convert solar energy. A photovoltaic monitoring system was designed to provide general parameters [12,13], e.g., energy conservation and stability, for a solar energy monitoring system with the selected parameters used for the photovoltaic cells and controller [14,15]. In [16] a method and system for non-invasive sensing and monitoring a processing system employed in semiconductor manu- 
facturing was disclosed. A utility model that discloses a photovoltaic greenhouse monitoring system based on a WSS is proposed in [17]. This system was comprised of wireless information collecting nodes with high reliability and easy to expand. Recently, the IOT (internet of thing) concept has become a very important technique applied to sensing, communication and networking. In the near future it will be applied mostly in monitoring and surveillance systems imbedded into internet environments $[18,19]$.

Green energy applications are gradually achieving an important position in industry development due to the shortage of renewable energy. Certainly, the issue of efficiency promotion in solar system to complete energy transformation becomes relatively very important. The requirement for a wireless surveillance system for "looking at" a charging system is necessary. This paper proposes a fresh idea to implement an uninterrupted solar surveillance system with a radio system. The proposed monitoring system is an ubiquitous system, i.e., it can be deployed at any location where a solar system is established. This system is designed to be initially established as a prototype model that can be commercialized after implementation and the measurements are completed. The measurement results of the implemented monitoring system provide useful results for the solar energy parameters to the operator who may not reside at the site via Internet protocol communication systems. The measurements are held at the campus around Dayeh University located in Central Taiwan at a 2.2 acre site [23].

The organization of this paper is as follows. Following the introduction section, the system implementation architecture is described in Section 2. The implementation results are shown in Section 3. Section 4 draws the study conclusions.

\section{Description of System Architecture}

The proposed solar energy monitoring system has three sub-systems, including the charging sub-system, the controlling sub-system and display sub-system, as illustrated in Figure 1, and Figures 2(a) and (b), respectively. The design rules are explained in this sub-section. Moreover over, the flow chart of the full system is shown in Figure 3.

\subsection{Charging Sub-System}

The charging sub-system gathers energy through the solar cells, that is, the energy will be charged by the solar cell (photovoltaic) system and the energy will be stored in a long consuming battery. Back-up charging will be provided by the city power system if the energy cannot be maintained at a threshold value by the solar system.

To maintain the system capable of running non-interrupted, certain signals will be captured through the system nodes utilizing RFID (radio frequency identification) techniques. The system is presented in Figure $\mathbf{2}$ in which components identified, solar cell plant, also called PV (photovoltaic) array cell (or solar cell panel) with 260 Watt, a DC 12-V charging battery, a battery with about 0.5 KVA power in the charging sub-system. A DC-toDC converter that pulls voltage up to about $\sqrt{2} \times 110 \mathrm{~V}$. This voltage is translated to about $115-\mathrm{V}$ AC voltage, which will be put into the city power line supplied by the power company. After the waveform shaper filters the energy generated from the charging sub-system, power is provided to all of the other sub-systems. By the way, the 0.5 KVA power charging battery is also providing full controlling sub-system described in the following with extra power. Thereafter, the target of an uninterrupted solar energy can be reached, since the main control system could self-sufficiency with the resources. Such implementation is embedded into the system shown in Figure 4. However, the power saving efficiency isn't calculated in this stage of the investigation.

\subsection{Controlling Sub-System}

The controlling sub-system, as shown in Figure 4, records and analyzes the data transmitted from the charging sub-system using an active embedded radio reader that reads the detected data passed from the tags. There will be tags attached in sub-system charging places where the active RFID system reader will read the effective data into the server via a serial port that communicates with a control server [20]. Thus, this sub-system is realized with hardware and soft ware components described as below:

The A/D (analog to digital) converter samples, quantizes and codes the received data. A cost effective matching micro processor in the A/D device ADC0804, which

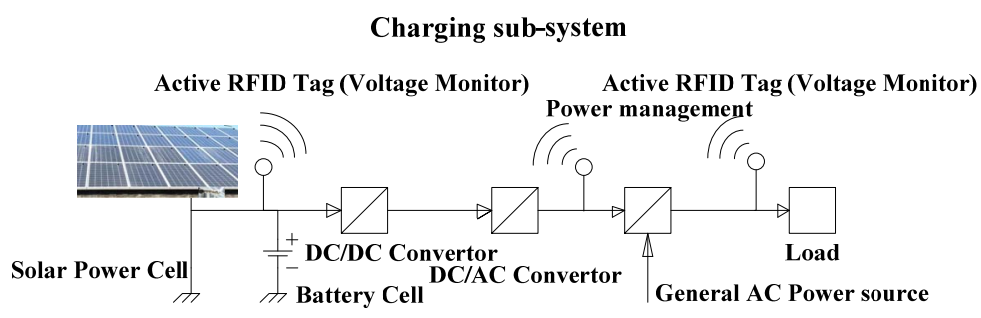

Figure 1. Block diagram of the charging sub-system. 


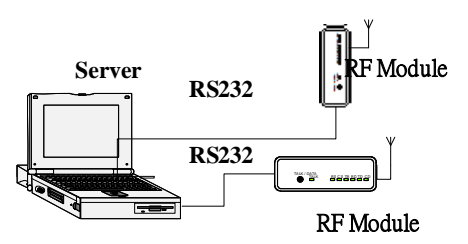

(a)

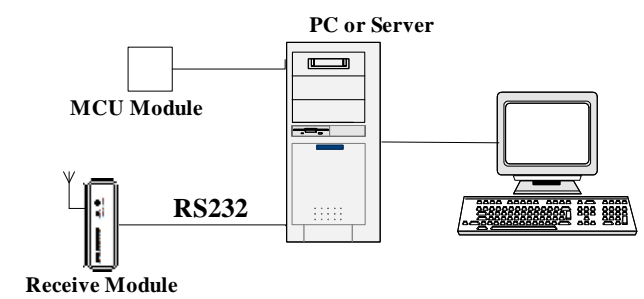

(b)

Figure 2. Block diagram of the display sub-system, (a) transmitting end; (b) receiving end.

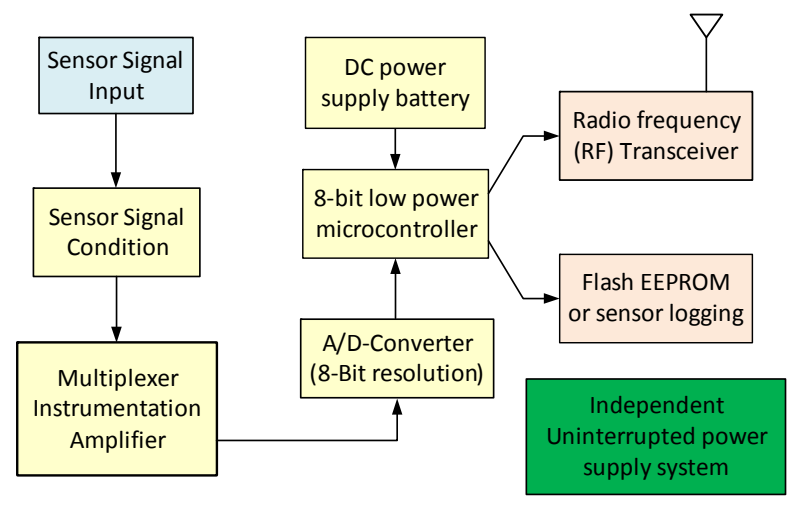

Figure 3. The flow chart of the full system.

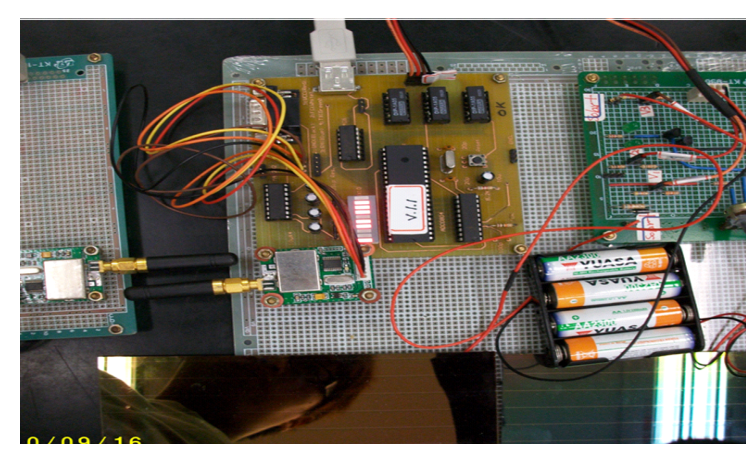

Figure 4. The solar energy monitoring prototype system.

is a serial product from Intersil Company with 8 bits precision, is chosen for the converter in this implementation. The ADC0804 specifications can be found in its data sheet [21]. There are 3 sample points designed to absorb alternatively the analog amplitude from the solar cell. The sampling rate can be adjusted by the crystal oscillator frequency of the MCU, hence, the transmission rate is adjustable. However, there still has an upper limitation rate depends on the specification of $\mathrm{A} / \mathrm{D}$ converter.
The core sub-system control techniques constrained by the micro-controller or micro-process in charge of all arithmetic and logic operations. Based on the usual reasons for choosing a micro processor, e.g., multi-functional, simple designing, low cost and easy to acquire the chip packaged and numbered "AT89C51 PC24" from the @ATMEL company has become the candidate for this sub-system [22]. Moreover, the specified PC board serial number 89C51 packaged in PDIP (plastic dual inline package) form was selected as the main chip designated for the main circuit. Another reason is the iron welding operation will easily become widespread in this area. It is known that the operating time clock of the 89C51 is generated from an internal oscillator (pin 18 and pin 19) with a frequency of $11.0592 \mathrm{MHz}$. Once the 89C51 baud rate is determined using a precision value generated from an embedded internal timer, the aforementioned operating frequency will have stability matched to the $9600 \mathrm{bps}$ (bit per second) operating baud rate of the RF (radio frequency) module which passes the solar cell messages. The 89C51 MODE 1, with 9600 band rate without parity check bit, 8 data bits and one stop bit, i.e., " $9600, N$, 8 , 1 " communication protocol, is operating in TH1 of internal timer 1 (timer 1 high bit). Moreover other operating modes are set with MODE 2 of 8 bit auto-loaded, SMODE $=0$ of single baud rate, hereafter, the right value of TH1 can be calculated as [21]

$$
T H 1=256-\frac{2^{S M O D} \times f_{o s C}}{386 \times\left(R_{B}\right)}
$$

where SMOD denotes the internal TIMER model type of 89C51, $f_{\text {osc }}$ represents the crystal oscillator frequency, $R_{B}$ is the baud rate in the communication port. For example, the internal timer 1 , TH1, will become as

$$
T H 1=256-\frac{2^{0} \times 11.0592 \times 10^{6}}{386 \times 9600}
$$

when 9600 and model 0 timer baud rate are selected. Several important pin assignments are described as follows. The pin number P3.0 and P3.1 of 89C51 PORT3 are assigned as the communicating to and receiving function from the RF module, respectively. On the other hand, P3.0 and P3.1 are correspondingly connected to the TX and RX of the RF module and the P3.2 and P3.3 of PORT3 are two pins for accepting external interruption. The 8 pins P0.0 to P0.7 of PORT0 are designated connecting to an aligned 8 LEDs for error monitoring. The data bits (DB7 to DB0) coming out from the A/D device ADC0804 are arranged in contact with P1.7 to PT1.0 of 89C51 PORT 1. There are 3 connectors P2.0, P2.1, P2.2 of PORT2 which are assigned to periodically (adjustable) absorb the sampled data from 3 reedy relays (DIA050000, 
$I_{d}=10 \mathrm{~mA}, V_{d}=5 \mathrm{~V}$, defined in the data sheet [21]) derived using 3 open collector inverters, respectively. The reedy relay operating time is $0.5 \mathrm{~ms}$ which is important for reacting to receive the signal from $\mathrm{A} / \mathrm{D}$ converter, i.e., the operation frequency is almost able to reach 800 Hz. PORT2 P2.3 and P2.4 are for $\overline{R D}$ and $\overline{W R}$ of ADC0804 to play as the signaling role when the data is read and written already, respectively. In contrast, P2.5 at PORT2 be linked to INTR of ADC0804 for informing the ready status which the A/D convert is completed. Furthermore, the advantage of RF transmission in MCU $89 \mathrm{C} 51$ is useful for this design due to its variable transmission rate, from 1200 to $19,200 \mathrm{~b} / \mathrm{s}$ which is helpful for the incremental the performance of data transfer. On the other hand, it's I/O ports is very Flexible for different transmission protocols, such as Bluetooth, ZigBee, Wifo, which can greatly improve the system performance.

Finally, the wireless transmission scenario is established using a $433 \mathrm{MHz}$ RF module equipped with transmission distance capability of 500 meters without obstacles. The overall specification can be checked at the Web page of [18]. The solar surveillance system prototype is designed with the detailed block diagram shown in Figure 2.

\subsection{Displaying Sub-System}

At this stage a script programmed with @Visual Basic software are applied to all functions for displaying the subsystems [7]. The captured data from the Tag components will be detected by the active reader and the complete information passed to the end server via the RF modem modules.

The total system is shown in Figure 5, implemented to integrate all sub-system for status display according to the data passed from the previous sub-system. The monitored values include the operating current, voltage, power, efficiency and storage capacity are analyzed and shown up in this sub-system. Hereafter, the total system operating efficiency can be tracked and tuned through the sub-system using the feedback signals. of the system management display panel is designed and shown in Figure 5. There is a communication protocol setting for adjusting the dual port parameters. The total count displays the gathered solar data records and there are 3 nodes for showing the voltage values including BV (battery voltage), SLV (solar with load voltage), and SWLV (solar without loading voltage) with the expression in hexadecimal format illustrated in Table 1. The error code column illustrates the corresponding error status occuring during the monitoring interval. A column named "data file content" displays the date and time information involved in status events. All necessary information about this solar energy system can be monitored through the panel set up.

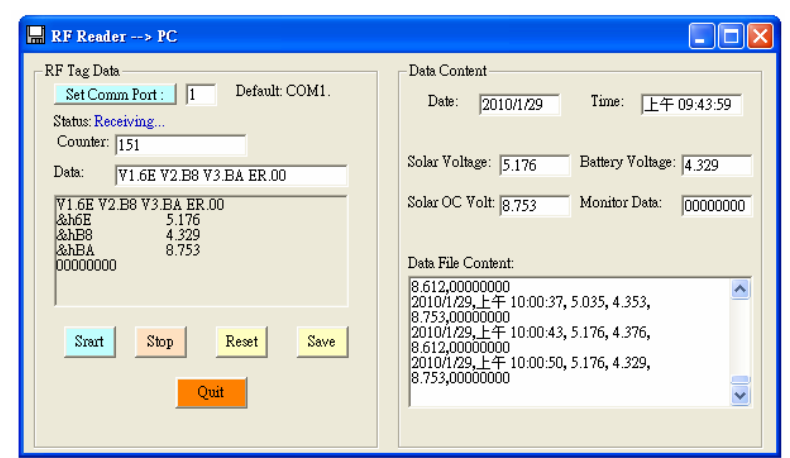

Figure 5. Collected data on the display sub-system.

Table 1. The collected data format.

\begin{tabular}{ccccccc}
\hline \multirow{2}{*}{ BV } & $\begin{array}{c}\text { Hex. } \\
\text { value }\end{array}$ & SLV & $\begin{array}{c}\text { Hex. } \\
\text { value }\end{array}$ & SWLV & $\begin{array}{c}\text { Hex. } \\
\text { value }\end{array}$ & $\begin{array}{c}\text { ERROR. } \\
\text { CODE }\end{array}$
\end{tabular}

For evaluating the lifetime of any sensor nodes deployed in the WSS environment some conditions are taken into account in the integrated sub-systems. All consumed energy is supported by the charging sub-system.

\section{Results and Simulations}

The prototype system and simulated implementation are finished using universal PC boards. The full integrated system is shown in Figure 4, wherein the illustration is marked. Some rough system parts are presented in Figure 4, The 16 counts receivered data will be transmitted to the server through the RF modules. The data format that comes from the reader is shown in Table 1. The information includes items described in the last 2.3 subsection sucessively shown in Figure 5. The most important elements in the monitoring system is the stability and realiability. It is known that interference generated from the environment is the critrical issue in RF transmission. The prototype system was verified using two experimental scenarios at the Dayeh university campus. The two experimental cases were set up using different RF transmission modules, i.e., the first using $433 \mathrm{MHz}$ and the second using $928 \mathrm{MHz}$. The simulated solar energy system and the monitoring system were placed at the front door of Dayeh University and inside the Lab room, respectively. The distance between these two places was about 200 meters. The measured voltage data included $\mathrm{BV}, \mathrm{SLV}$, and SWLV, gathered during the afternoon hours 12:00 to 17:00 PM. The aforementioned experiment exhibited a large amount of channel fading, which included large scale fading (huge academy building, big tree), and small scale fading (pedestrians). Because the class was dismissed at the noon time 12:00 to PM 12:30 interval, the inturruptions were caused mostly by people moving around the experimental area. This phenomenon was satisfactory and expected. The RF modules defini- 
tely interfered with the fading coming out of the transmission channel. It is an original idea of power management in solar cell implemented in this article. Since the real-time data of the solar cell is transmitted by RF protocol which differs from the one shown in [2,3]. However, it should be pointed out the shortage in distance of our designed, that is, the separation between the solar cell platform and the mornitoring terminal will be constrained due to the RF protocol. Furthermore, for confirmation there are experimental results shown in Figure 6 obtained from the simulation which is evaluated with the assessment of university campus. The distance is about 10 meter, a pair of RF transmission modules $928 \mathrm{MHz}$ is applied. There are 3 simulated curved shown in Figure 6 in which the results from reveiving the digitalized solar cell data, therein, the curve with little triangle, the plot with little square, and the graph with little circle represent solar voltage with non-loading, solar voltage with full-loading, and the battery voltage, respectively. All the curves were collected during the period of AM 11:00 to PM 2:30 which is scaled as $\mathrm{Y}$ axis (time axis) and $\mathrm{X}$-axis represents the amplitude of voltage. It is reasonable to understand that the curve with little triangle is eaasily interrupted by shadeling, for example, the building, the pedestrians, or some tree. Besides, the other two kinds of measured voltages are much more stable. These facts significantly prove that the developed monitoring system in this article is definitely a new design. The most difference of results between this development and the others is that the wireless communication technologies are applied.

\section{Current \& Future Developments}

The results from the implementation were completed as illustrated with experimental measurements. The RF modules, which can be alternatively replaced with Bluetooth, were adopted to transmit the necessary solar energy system information wirelessly. The WSS concept was also applied in the data gathering implementation from different places where solar energy systems could be installed. To avoid the severe shadowing effect from different transmission protocols such as ZigBee, GPRS, and WiMax, these technologies will be tested in the future in a suitable environment. The RFID techniques can be fused into the implementation for secure purposes. At the Dayeh university campus we adopted two experimental deployments, which were set up with different RF transmission modules, $433 \mathrm{MHz}$ and $928 \mathrm{MHz}$. The simulated solar energy system and the monitoring system were placed at the front door of Dayeh University and inside the Lab room, respectively. The distance between these two places was about 200 meters. The solar voltages with full and non-full loder are measured, also the battery voltage. During the interval 12:00 to 17:00 PM. The

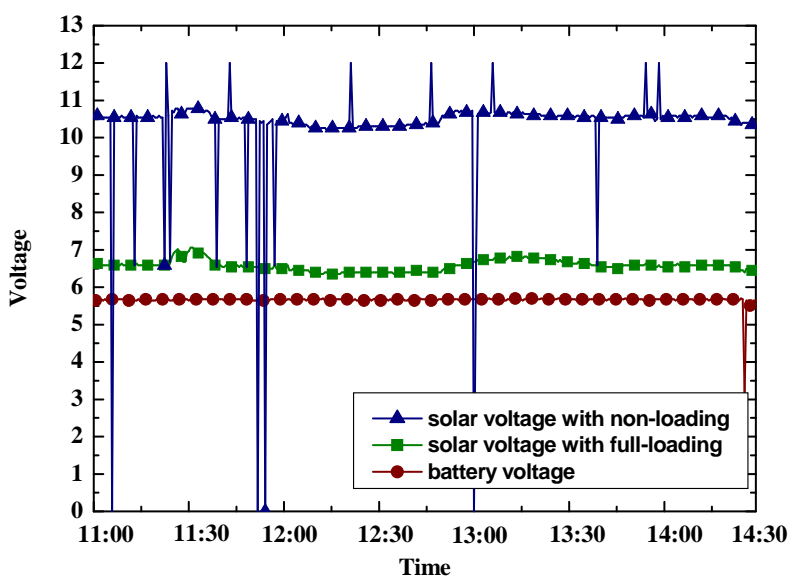

Figure 6. Experienced voltage curves with indoor of a classroom at engineering building in Dayeh university campus to outdoor using $928 \mathrm{MHz}$ RF transmission type.

experiment exhibited a large amount of channel fading, which included large scale fading (huge academy building, big tree), and small scale fading (pedestrians). Because the class was dismissed at the noon time 12:00 to PM 12:30 interval, the inturruptions were caused mostly by people moving around the experimental area. The detail envirnment can look for the Web page of the Dayeh university [23].

\section{Acknowledgements}

The author would like to thank the anonymous reviewers and the editor for their helpful comments that considerably improved the quality of this paper.

\section{REFERENCES}

[1] N. T. Iea, "Energy Efficiency and Renewable Energy-A Key to a Better Tomorrow," ISO Open Session, Cape Tow, 17 September 2009.

[2] G. Mikael and L. Tomas, “An Energy Efficient Method for Communication between a Wireless Sensor Network and an Industrial Control System,” WO Patent No. 2011 154051A1, 2011.

[3] Z. Y. Yao and D. Yoonmee, "Wireless Sensor Network and Adaptive Method for Monitoring the Security Thereof,” US Patent 8116243, 2012.

[4] W. X. Wang, J. W. Sung and D. Y. Kim, “Complex Event Processing in EPC Sensor Network Middleware for Both RFID and WSN," 2008 11th IEEE Interference Symposium on Communications, Networking, and Broadcasting, Lapland, 8-11 September 2008, pp. 165-169.

[5] X. Shan and J. Tan, "Mobile Sensor Deployment for a Dynamic Cluster-Based Target Tracking Sensor Network,” 11th IEEE Interference Symposium on Communications, Networking, and Broadcasting, New York, 2005, pp. 14521457.

[6] T. Kim, J. Lee, S. Lee and A.-C. Park, "Performance 
Evaluation of RTLS Based on Active RFID Power Measurement for Dense Moving Objects," IEICE Transactions on Communications; Vol. E92-B, No. 4, 2009, pp. 14221425.

[7] Wireless Sensor Network Center, "Mobile Network Supported Wireless Sensor Network Services,” 2007. http://www.wsnc.ntu.edu.tw/

[8] B. Christof and M. S. Brian, "Modular Monitor Service for Smart Item Monitoring," US Patent No. 8131838, 2012.

[9] B. Visweswaran and A. R. Kulkarni, "Green Luxury, Technology and Solutions for Energy Management," IEEE Conferences (ICM), Marrakech, 19-22 December 2009, pp. 138-140.

[10] D. Niyato, E. Hossain and V. K. Bhargava, "Wireless Sensor Networks with Energy Harvesting Technologies: A Game-Theoretic Approach to Optimal Energy Management," IEEE Wireless Communications, 2007, pp. 9096. doi:10.1109/MWC.2007.4300988

[11] http://www.advantech.gr/ia/3175.htm

[12] J. Hou and Y. Gao, "Greenhouse Wireless Sensor Network Monitoring System Design Based on Solar Energy," International Conference on Challenges in Environmental Science and Computer Engineering, Wuhan, 6-7 March 2010, pp. 475-479.

[13] P. Ray and P. Raju, “Apparatus and Methods for Managing Power Capacity in Data Centers Using a Wireless Sensor Network,” US Patent No. 0054527, 2012.

[14] B. Greg and C. Matt, "Photovoltaic System with Man- aged Output and Method of Managing Variability of Output from a Photovoltaic System,” US Patent No. 0224831, 2011.

[15] L. Bin, C. Yanbo and Z. Lihua, "The Design of Photovoltaic Monitoring System,” Power Electronics Systems and Applications (PESA), Vol. 1, No. 4, 2011, pp. 1-4.

[16] K. Sanjeev and S. Kenji, "Method of Monitoring a Semiconductor Processing System Using a Wireless Sensor Network,” US Patent No. 8026113, 2011.

[17] X. Zhong and F. Wang, "Photovoltaic Greenhouse Monitoring System Based on WSN (Wireless Sensor Network)," CN Patent No. 202041835, 2011.

[18] G. Wu, S. Talwar, K. Johnsson, N. Himayat and K. D. Johnson, "M2M: From Mobile to Embedded Internet," IEEE Communications Magazine, Vol. 49, No. 4, 2012, pp. 36-44.

[19] Dayeh University Web Site, 2012. http://www.dyu.edu.tw/english/1100.php

[20] Intersil Americas Inc., "ADC0803, ADC0804 8-Bit, Microprocessor-Compatible, A/D Converters Data Sheet," FN3094, 2002.

[21] Atmel Corporation, "8-Bit Microcontroller with 4K Bytes Flash AT89C51,” 2000. http://www.atmel.com/atmel/acrobat/doc0265.pdf

[22] ICtec Company, “433MHz, 200 Meter RF Module Serial No. A06-0016.” http://www.icci.com.tw

[23] Dayeh University Web Site, 2012. http://www.dyu.edu.tw/english/ 